\title{
Development of Pilot Scale System for Production of Polyamide-6 Fibers Grafted with Polymethacrylic Acid for Ion Exchange Applications
}

\author{
S.E. Shalaby*, S. M. Abo El-Ola, N. G. Balakocy, M. K. Beliakova, R. A. Abas \\ Textile Research Division, National Research Centre, Dokki, Cairo, Egypt. (Scopus \\ Affiliation ID: 60014618)
}

\begin{abstract}
$\mathbf{R}$ EMOVING the hazardous metals from wastewater especially industrial drainage is very important for preventing health problems in human beings, plants and animals. The ion exchanger has constructed as primary line of safeguards for that goal. In this study grafted polyamide-6 (PA-6) fibers with polymethacrylic acid (PMAA) was used as materials for the ion exchangers. Grafting was achieved under required time and temperature and the factors affecting the graft process were studied. The efficiency of the grafted fibers for adsorption of $\mathrm{Cu}^{2+}, \mathrm{Pb}^{2+}$ and $\mathrm{Cr}^{6+}$ was evaluated. The effect of adsorption parameters such as $\mathrm{pH}$, duration of adsorption, initial ion concentration and the adsorption temperature on the degree of extraction ( $\mathrm{R} \%$ ) and adsorption capacity (SC, mg/g) was studied. Optimization the conditions for the preparation on laboratory scale, adjustment and adaptation of these conditions to suit the production of these grafted fibers on pilot scale was accomplished.
\end{abstract}

Keywords: Polyamide-6, Grafting, Fibers, Ion exchanger.

\section{Introduction}

In order to remove the hazardous metals from wastewater especially industrial drainage, there are common methods used for that purpose such as air stripping reverse osmosis, flocculation, and sedimentation, soil flushing/washing chelating, chemical precipitation, reduction, and ion exchange. Ion exchange has constructed as primary line of safeguards and especially for those which cannot be removed by other methods. Consequently, the materials used for ion exchange are performing a great role in pollution prevention.

These materials include chemisorptions fibers and fabrics with a developed characteristics surface. Scientific researches discuss different methods for manufacturing of the ion- exchange materials such as creation of functional active groups in the fiber polymers via reactions in polymer chains [1-2], and grafting of fibers with inorganic and organic monomers with subsequent reactions in the grafted chains [3-6]. The fibers could be used as material for different purpose such as ion- exchangers, complexions, oxidants, reluctant and catalysts [7]. The fibrous materials have a wide range of implementations such as the separation of earth rare elements [8], air purification from contaminates such as acids, acidic oxides, alkalis, ammonia and amines, chlorine etc [7], and water purification [8].

The ion-exchange fibers are manufactured either by polymer analogous conversion of the reactive groups in the macromolecules of the fibers [9], or by grafting of various vinyl monomers, containing different function groups, on to the surface of fiber $[7,10]$.

PA-6 fibers are one of the most remarkable manmade fibers utilized in the textile industry. As well as, it could be used as materials for ion-exchangers. The major number of PA fibers were prepared by grafting with different vinyl monomers such as methyl vinyl pyridine (MVP), glycidyl methacrylate (GMA), styrene (ST), acrylonitrile (AN), methacrylic acid (MAA) and dimethyl amino ethymethacrylate

*Corresponding author e-mail: samyshalaby40@yahoo.com

DOI: 10.21608/ejchem.2018.3631.1301

C2017 National Information and Documentation Center (NIDOC) 
(DMAEMA [11-14]. The reactive groups of these vinyl monomers pave the way for subsequent polymeranglous transformation in the grafted chains resulting in creation of new functional groups in the side chain of the copolymer which have been used for incorporation of sorptionactive groups in the fibers.

The most methods used for grafting the vinyl monomers onto PA fibers have limited usages because of low rate of grafting reaction and homopolymer formation. It is well known that the ion exchange capacity is one of the basic parameters for selecting the ion exchangers. Thus, the using preparation method for grafting should ensure that the grafting reaction will proceed with high rate and without homopolymer formation. It has been reported that, these requirements could be fulfilled only when PA fibers contain functional groups that are capable of forming a complex with the initiator on the fibers. Further decomposition of the obtained complex leads to the formation of free radicals on the fibers thus facilitating direct grafting with high rate and without homopolymer formation. It is well known that quaternary ammonium groups (QAG) can form complexes with peroxides. This was successfully used for grafting PA-6 fibers with different vinyl monomers [15-32].

The preparation of ion- exchangers based on grafted PA- 6 nonwoven fabrics is one of the new fields of application of PA-6 fibers. Optimal conditions for the preparation on laboratory scale are available. Adjustment and adaptation of these conditions to suit the production of these nonwoven fabrics on pilot scale is the main target of this work. Here, we represent the results of grafting PA-6 fibers containing antimicrobial substance (AS) with MAA using $\mathrm{Cu}^{2+}-\mathrm{K}_{2} \mathrm{~S}_{2} \mathrm{O}_{8}$ redox system and their application as ion exchanger for waste water treatment.

\section{Experimental}

\section{Materials}

- PA-6 used in this study was in the form of fibers (denier =14). kindly supplied by Masr Company, Elmehala, Egypt. The fibers were scoured at $80^{\circ} \mathrm{C}$ for 45 minutes, with solution containing $2 \mathrm{~g} / \mathrm{l}$ nonionic detergent, washed with water, squeezed and finally air dried.

- Methacrylic acid (MAA), potassium persulphate $\left(\mathrm{K}_{2} \mathrm{~S}_{2} \mathrm{O}_{8}\right)$, and copper sulphate $\left(\mathrm{CuSO}_{4} \cdot 5 \mathrm{H}_{2} \mathrm{O}\right)$ were all of pure grade chemicals

- Antimicrobial substance (AS) under trade name " katamine " was in the form of $50 \%$ aqueous solution (Russia).

\section{Methods}

Fixation of AS on PA-6 Fibers

The treatment of PA-6 fabrics with AS was carried out using a high temperature high pressure laboratory dyeing machine. Required amounts of AS solutions $(\mathrm{pH}=11)$ were placed in stainless-steel bowls, PA-6 samples were immersed in the solutions, and the sealed bowls were rotated in a closed bath containing ethylene glycol at the desired temperature $\left(90^{\circ} \mathrm{C}\right)$. The material-to-liquor ratio (M: L) was 1:50. The bath temperature increased at rate of $2^{\circ} \mathrm{C} / \mathrm{min}$. After the predetermined durations, the samples were removed from the bath, rinsed repeatedly with distilled water and allowed to dry in the open air.

\section{Grafting of PA-6 Fibers with MAA}

PA-6 fibers samples containing AS were treated with $2.0 \%$ aqueous solution of $\left(\mathrm{K}_{2} \mathrm{~S}_{2} \mathrm{O}_{8}\right)$ at room temperature. The samples were then removed, washed thoroughly with distilled water, squeezed and dried at room temperature. The samples retained some persulphate which was determined iodometrically. Pretreated samples were introduced into stopper Erlenmeyer flask containing water, MAA and copper sulfate. The flask was kept in water thermostat at prescribed temperature $\left(90^{\circ} \mathrm{C}\right)$ and shaken occasionally during the reaction period. After an elapsed time, the reaction mixture was filtered and the residue was washed with water, dried in an oven at $105^{\circ} \mathrm{C}$ for $2.0 \mathrm{~h}$. The dried sample was then repeatedly soxhlet extracted with methanol to remove the homopolymer, dried again as previously indicated and weighed. The percentage of grafted polymer was calculated as follows:

$$
\% \text { Graft yield }=\frac{\mathrm{P}-\mathrm{P}_{\mathrm{o}}}{\mathrm{P}_{\mathrm{o}}} \times 100
$$

Where: $\mathrm{P}$ is the dry weight of grafted sample, and $P_{0}$ is the dry weight of un-grafted sample.

\section{Adsorption Procedure}

Dried chemically modified PA-6 fiber grafted with PMAA samples (in salt form) (0.2g) were added in $100 \mathrm{ml}$ Erlenmeyer including volumes 60 $\mathrm{ml}$ of each metal ion solution (80 ppm) adjusted to the desired $\mathrm{pH}$. The content was shaken at 200 $\mathrm{rpm}$ for a predetermined period of time at $30^{\circ} \mathrm{C}$ 
using horizontal shaking water bath. At the end of the experiment the samples were filter and the ion concentration of the filtrate was analyzed with an atomic adsorption spectrophotometer.

The adsorption amount was calculated as follow: the degree of extraction ( $\mathrm{R}, \%)$ and sorption capacity $(\mathrm{SC}, \mathrm{mg} / \mathrm{g}$ ) were calculated with the following equations:

$$
\begin{aligned}
& \mathrm{R}=\left[\left(\mathrm{C}_{\mathrm{o}}-\mathrm{C}_{\mathrm{i}}\right) / \mathrm{C}_{\mathrm{o}}\right] \times 100 \\
& \mathrm{SC}=\left[\left(\mathrm{C}_{\mathrm{o}}-\mathrm{C}_{\mathrm{i}}\right) \times \mathrm{V}\right] / \mathrm{m}
\end{aligned}
$$

Where: $\mathrm{C}_{0}$ is the initial concentration of the investigated metal ion in the solution, $\mathrm{mg}$ /liter; $\mathrm{C}_{\mathrm{i}}$ is the concentration of investigated metal ion solution, $\mathrm{mg}$ /liter; $\mathrm{V}$ is the volume of solution, (1); $\mathrm{m}$ is the mass of the sorbent, $\mathrm{g}$.

\section{Results and Discussion}

Grafting PA-6 Fibers with MAA

The graft copolymerization of MAA onto PA-6 fibers containing AS in the presence of $\mathrm{K}_{2} \mathrm{~S}_{2} \mathrm{O}_{2}-\mathrm{Cu}^{2+}$ as redox initiating system was carried out on laboratory scale. The factors that affect the graft add-on were investigated. The optimum conditions for grafting of PA-6 fibers were discovered

The effect of different reaction conditions on the graft yield

\section{Effect of cupric ion concentration}

The effect of $\mathrm{CuSO}_{4} .5 \mathrm{H}_{2} \mathrm{O}$ concentrations on the extent and rate of grafting was investigated. The data of Tables 1 and 2 revealed that the, grafting reaction can proceed either in presence or the absence of $\mathrm{Cu}^{2+}$ in the reaction medium. But in this case the graft yield did not exceed $11.5 \%$ after carrying reaction for $60 \mathrm{~min}$. at $90^{\circ} \mathrm{C}$. The addition of $\mathrm{Cu}^{2+}$ in the reaction medium gives rise to an outstanding increase in both the graft yield and grafting rate $\left(R_{p}\right)$. This increase depends on the $\mathrm{Cu}^{2+}$ concentration. It was found that the maximum graft yield (55\%) on Nylon-6 fibers is obtained at a concentration of $1.25 \times 10^{-3} \mathrm{~mol} / \mathrm{l}$ of $\mathrm{CuSO}_{4} .5 \mathrm{H}_{2} \mathrm{O}$. Thereafter, the graft yield decreases as the $\mathrm{Cu}^{2+}$ ions concentration increases.

Kinetic investigation (Table 3, Fig. 1) of the dependence of the rate of grafting $\left(\mathrm{R}_{\mathrm{p}}\right)$ of MAA on PA-6 fibers containing AS revealed that, $R_{p}$ is proportional to 0.45 power of $\mathrm{Cu}^{2+}$.

\section{Effect of MAA concentration}

The effect of MAA concentrations on the extent and rate of grafting was investigated
(Tables 4-5). The monomer concentration was in the range from $0.17 \mathrm{~mol} / 1-2.32 \mathrm{~mol} / \mathrm{l}$. It was clear from the data that, the maximum extent $(55.0 \%)$ and rate of grafting $\left(3.45 \times 10^{-5} \mathrm{~mol}^{-1}\right.$. $\mathrm{sec}^{-1}$ ) are attaining at $5.8 \times 0^{-1} \mathrm{~mol} / 1$ concentration of MAA. Further increase in MAA concentration is accompanied with a decrease in the grafting extent. Kinetic investigation presented in Table 6 and Fig. 2 appeared that, the rate of grafting $\left(R_{p}\right)$ is proportional to 0.89 power of [MAA].

\section{Effect of Temperature}

The effect of temperature on the extent and rate of grafting of MAA on PA- 6 fibers, containing AS was investigated. It is clear from Tables 7-8 that, both the extent and $\mathrm{Rp}$ increase as the reaction temperature increases from $75^{\circ} \mathrm{C}$ to $90^{\circ} \mathrm{C}$. Based on data presented in Table 8 and in Fig. 3, the apparent activation energy of MAA grafting onto PA-6 fibers containing AS was calculated and was found to be $102.0 \mathrm{~K} \mathrm{~J} \mathrm{~mol}^{-1}$.

\section{Effect of $\mathrm{K}_{2} \mathrm{~S}_{2} \mathrm{O}_{8}$ Concentration}

The effect of reaction time on the extent and rate of grafting onto PA-6 fibers containing AS over a range of $1.02 \times 10^{-4}$ to $2.20 \times 10^{-4} \mathrm{~mol} / 1$ $\mathrm{K}_{2} \mathrm{~S}_{2} \mathrm{O}_{8}$ was investigated. It was found that, the graft add- on and rate of grafting $\left(\mathrm{R}_{\mathrm{p}}\right)$ increase with the increase of $\mathrm{K}_{2} \mathrm{~S}_{2} \mathrm{O}_{8}$ concentration and the reaction time (Tables 9-10). The maximum extent of grafting was obtained at $2.20 \times 10^{-4} \mathrm{~mol} / 1$ $\mathrm{K}_{2} \mathrm{~S}_{2} \mathrm{O}_{8}$ in the reaction medium after 60 minutes.

Kinetic investigation (Fig. 4) revealed that, $\mathrm{R}_{\mathrm{p}}$ is proportional to 0.59 power of $\mathrm{K}_{2} \mathrm{~S}_{2} \mathrm{O}_{8}$ concentration.

Based on the above mentioned kinetic investigation of the effect of different factors on the grafting reaction of MAA onto PA-6 fibers containing AS one can conclude that, the overall $\mathrm{R}_{\mathrm{p}}$ is proportional to $\left[\mathrm{Cu}^{2+}\right]^{0.45}[\mathrm{MAA}]^{0.89}$ $\left[\mathrm{K}_{2} \mathrm{~S}_{2} \mathrm{O}_{8}\right]^{\mathrm{p} .59}$

\section{Pilot scale production of PA-6 fibers grafted with PMAA}

The above mentioned investigations open the way to select the technically feasible option for the production of the grafted PA-6 fibers with vinyl monomers on pilot scale.

It should be stated that, the grafted PA-6 fibers will be converted to nonwoven ion- exchange fabrics. Therefore, its fiber ability should be preserved. This can be obtained by controlling the percentages of grafted polymer on the surface of fibers. The latter can be achieved by the variation 
of the weights of both of monomer and fibers in the reaction medium. Table 11 illustrates, for example, the effect of the relation between the weight of MAA and the weight of fibers on the amount of grafted PMAA on the PA-6 fibers.
Based on the data listed in Table 11 one can conclude that, the optimum ratio between the weight of the monomer to the fibers weight which can preserve the fiber ability is ranging from $0.5: 1$ to $0.75: 1$.

TABLE 1. Effect of Cupric ions concentration on the graft yield \% of MAA onto PA-6 fibers.

\begin{tabular}{cc}
\hline $\left.\mathbf{C u S O}_{\mathbf{4}} . \mathbf{5} \mathbf{H}_{\mathbf{2}} \mathbf{O}\right] \times \mathbf{1 0}^{-3} \mathbf{~} \mathbf{~ o l} / \mathbf{l}$ & Graft Yield \% \\
\hline 0.00 & 11.5 \\
0.05 & 17.8 \\
0.125 & 22.6 \\
0.187 & 29.6 \\
0.25 & 45.2 \\
0.75 & 50.3 \\
1.25 & 55.0 \\
2.50 & 34.2 \\
3.75 & 28.8 \\
\hline
\end{tabular}

Reaction Conditions:

[MAA], $5.8 \times 10^{-1} \mathrm{~mol} / 1 ;\left[\mathrm{K}_{2} \mathrm{~S}_{2} \mathrm{O}_{8}\right], 2.20 \times 10^{-4} \mathrm{~mol} / \mathrm{l} ;[\mathrm{AS}], 1.63 \mathrm{mg} / \mathrm{g}$ fibers; Reaction Temperature, $90^{\circ} \mathrm{C}$; Reaction Time, 60 min.; M: L, 1:50.

TABLE 2. Effect of Cupric ions concentration on the rate of grafting (Rp) of MAA onto PA-6 fibers.

\begin{tabular}{|c|c|c|c|c|c|c|c|}
\hline \multirow{2}{*}[\mathrm{CuSO}_{4}.5\mathrm{H}_{2}\mathrm{O}]{$\times 10^{-3} \mathrm{~mol} / \mathrm{l}$} & \multicolumn{5}{|c|}{ Reaction Time (min.) } & & \\
\hline & 10 & 20 & 30 & 45 & 60 & & \\
\hline 0.05 & 4.20 & 6.50 & 9.70 & 12.3 & 14.2 & \multirow{4}{*}{$0^{\circ}$} & \multirow{4}{*}{ 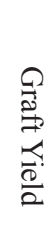 } \\
\hline 0.125 & 6.50 & 8.50 & 12.8 & 19.6 & 22.6 & & \\
\hline 0.25 & 10.5 & 13.7 & 22.9 & 35.4 & 41.2 & & \\
\hline 1.25 & 17.8 & 28.1 & 37.2 & 46.6 & 55.0 & & \\
\hline
\end{tabular}

Reaction Conditions:

[MAA], $5.8 \times 10^{-1} \mathrm{~mol} / 1 ;\left[\mathrm{K}_{2} \mathrm{~S}_{2} \mathrm{O}_{8}\right], 2.20 \times 10^{-4} \mathrm{~mol} / 1 ;$ [AS], $1.63 \mathrm{mg} / \mathrm{g}$ fibers; Reaction Temperature, $90^{\circ} \mathrm{C}$; M:L, 1:50.

TABLE 3. Dependence of the rate of grafting (Rp) of MAA onto PA-6 fibers on the $\mathrm{Cu}^{+2}$ concentration.

\begin{tabular}{|c|c|c|c|c|c|c|c|c|}
\hline \multirow{2}{*}{$\begin{array}{c}{[\mathrm{Cu}] \times \mathbf{x 1 0}^{-3}} \\
\mathrm{~mol} / \mathrm{l}\end{array}$} & \multirow{2}{*}{$\begin{array}{c}\mathrm{Ln} \\
{[\mathrm{Cu}]} \\
\end{array}$} & \multirow{2}{*}{$\begin{array}{c}\text { Ln } \\
{[\mathrm{Cu}]+10}\end{array}$} & \multicolumn{3}{|c|}{ Graft Yield } & \multirow{2}{*}{$\begin{array}{c}\mathrm{Rp} \\
\text { mol.l }^{-1} \text {.sec }^{-1}\end{array}$} & \multirow{2}{*}{ LnRp } & \multirow{2}{*}{$\begin{array}{c}\text { Ln } \\
\text { Rp+12 }\end{array}$} \\
\hline & & & $\%$ & gr. & $\mathrm{mol} / \mathrm{l}$ & & & \\
\hline 0.05 & -9.90 & 0.10 & 4.2 & 0.021 & $4.88 \times 10^{-3}$ & $8.14 \times 10^{-6}$ & -11.72 & 0.28 \\
\hline 0.125 & -8.98 & 1.02 & 6.5 & 0.033 & $7.67 \times 10^{-3}$ & $1.28 \times 10^{-5}$ & -11.27 & 0.73 \\
\hline 0.25 & -8.30 & 1.70 & 10.5 & 0.053 & $1.22 \times 10^{-2}$ & $2.03 \times 10^{-5}$ & -10.80 & 1.20 \\
\hline 1.25 & -6.70 & 3.30 & 17.8 & 0.089 & $2.07 \times 10^{-2}$ & $3.45 \times 10^{-5}$ & -10.27 & 1.73 \\
\hline
\end{tabular}

Reaction Conditions:

[MAA], $5.8 \times 10^{-1} \mathrm{~mol} / 1 ;\left[\mathrm{K}_{2} \mathrm{~S}_{2} \mathrm{O}_{8}\right.$ ], $2.20 \times 10^{-4} \mathrm{~mol} / 1 ;[\mathrm{AS}], 1.63 \mathrm{mg} / \mathrm{g}$ fibers; Reaction Temperature, $90^{\circ} \mathrm{C}$; Reaction Time, 10 min.; M:L, 1:50.

Egypt. J. Chem. 61, No. 6 (2018) 


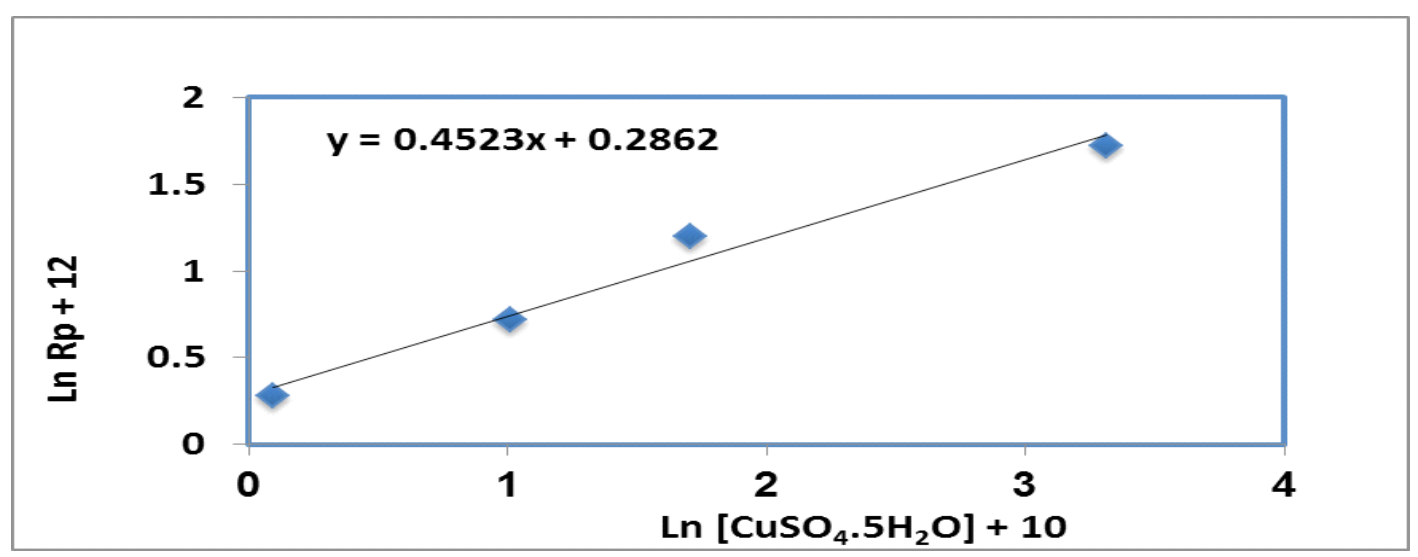

Fig.1. Logarithmic correlation between the rate of grafting (Rp) of MAA onto PA-6 fibers on the $\mathrm{Cu}^{+2}$ concentration. Reaction Conditions:

[MAA], $5.8 \times 10^{-1} \mathrm{~mol} / 1 ;\left[\mathrm{K}_{2} \mathrm{~S}_{2} \mathrm{O}_{8}\right], 2.20 \times 10^{-4} \mathrm{~mol} / 1 ;[\mathrm{AS}], 1.63 \mathrm{mg} / \mathrm{g}$ fibers; Reaction Temperature, $90^{\circ} \mathrm{C}$; Reaction Time, 10 min.; M:L,1:50.

TABLE 4. Effect of MAA concentration on the graft yield \% onto PA-6 fibers.

\begin{tabular}{cc}
\hline [MAA],mole/ $\mathbf{l}$ & Graft Yield (\%) \\
\hline 0.17 & 11.8 \\
0.29 & 26.8 \\
0.44 & 36.2 \\
0.58 & 55.0 \\
0.87 & 34.5 \\
1.16 & 31.2 \\
1.45 & 26.7 \\
1.74 & 20.1 \\
2.23 & 19.5 \\
\hline
\end{tabular}

Reaction Conditions:

$\left[\mathrm{CuSO}_{4} .5 \mathrm{H}_{2} \mathrm{O}\right], 1.25 \times 10^{-3} \mathrm{~mol} / \mathrm{l} ;\left[\mathrm{K}_{2} \mathrm{~S}_{2} \mathrm{O}_{8}\right], 2.20 \times 10^{-4} \mathrm{~mol} / \mathrm{l} ;[\mathrm{AS}], 1.63 \mathrm{mg} / \mathrm{g}$ fibers; Reaction Temperature, $90^{\circ} \mathrm{C}$; Reaction Time, 60 min.; M:L, 1:50.

TABLE 5. Effect of MAA concentration on the rate of grafting (Rp) of MAA onto PA-6 fibers.

\begin{tabular}{|c|c|c|c|c|c|c|c|}
\hline \multirow{2}{*}[\mathrm{MAA}]{$\times 10^{-1}, \mathrm{~mol} / \mathbf{l}$} & \multicolumn{5}{|c|}{ Reaction Time (min.) } & & \\
\hline & 10 & 20 & 30 & 45 & 60 & & \\
\hline 0.17 & 5.80 & 7.90 & 10.8 & 11.3 & 11.8 & \multirow{4}{*}{$0^{\circ}$} & \\
\hline 0.29 & 10.7 & 12.1 & 15.4 & 25.2 & 26.8 & & 常 \\
\hline 0.44 & 13.8 & 19.6 & 26.3 & 31.5 & 36.2 & & $\frac{\tilde{0}}{2}$ \\
\hline 0.58 & 17.8 & 28.1 & 37.2 & 46.6 & 55.0 & & \\
\hline
\end{tabular}

Reaction Conditions:

$\left[\mathrm{CuSO}_{4} \cdot 5 \mathrm{H}_{2} \mathrm{O}\right], 1.25 \times 10^{-3} \mathrm{~mol} / 1 ;\left[\mathrm{K}_{2} \mathrm{~S}_{2} \mathrm{O}_{8}\right], 2.2 \times 10^{-4} \mathrm{~mol} / \mathrm{l} ;[\mathrm{AS}], 1.63 \mathrm{mg} / \mathrm{g}$ fibers; Reaction Temperature, $90^{\circ} \mathrm{C} ; \mathrm{M}: \mathrm{L}$, 1:50. 
TABLE 6. Dependence of the rate of grafting (Rp) of MAA onto PA-6 Fibers on the monomer concentration.

\begin{tabular}{|c|c|c|c|c|c|c|c|c|}
\hline \multirow{2}{*}{$\begin{array}{c}\text { [MAA] } \\
\text { mol/l }\end{array}$} & \multirow{2}{*}{$\begin{array}{c}\text { Ln } \\
\text { [MAA] }\end{array}$} & \multirow{2}{*}{$\begin{array}{c}\text { Ln } \\
{[\mathbf{M A A}]+2}\end{array}$} & \multicolumn{3}{|c|}{ Graft Yield } & \multirow{2}{*}{$\begin{array}{c}\mathrm{Rp} \\
\text { mol. }^{-1} . \mathrm{sec}^{-1}\end{array}$} & \multirow{2}{*}{ Ln Rp } & \multirow{2}{*}{$\begin{array}{c}\text { Ln } \\
\text { Rp+12 }\end{array}$} \\
\hline & & & $\%$ & gr. & $\mathrm{mol} / \mathrm{l}$ & & & \\
\hline 0.17 & -1.77 & 0.23 & 5.8 & 0.029 & $6.74 \times 10^{-3}$ & $1.12 \times 10^{-5}$ & -11.40 & 0.60 \\
\hline 0.29 & -1.24 & 0.76 & 10.7 & 0.054 & $1.24 \times 10^{-2}$ & $2.07 \times 10^{-5}$ & -10.78 & 1.22 \\
\hline 0.44 & -0.82 & 1.18 & 13.8 & 0.069 & $1.60 \times 10^{-2}$ & $2.67 \times 10^{-5}$ & -10.53 & 1.47 \\
\hline 0.58 & -0.54 & 1.45 & 17.8 & 0.089 & $2.07 \times 10^{-2}$ & $3.45 \times 10^{-5}$ & -10.27 & 1.73 \\
\hline
\end{tabular}

Reaction Conditions:

$\left[\mathrm{CuSO}_{4} .5 \mathrm{H}_{2} \mathrm{O}\right], 1.25 \times 10^{-3} \mathrm{~mol} / \mathrm{l} ;\left[\mathrm{K}_{2} \mathrm{~S}_{2} \mathrm{O}_{8}\right], 2.20 \times 10^{-4} \mathrm{~mol} / \mathrm{l} ;[\mathrm{AS}], 1.63 \mathrm{mg} / \mathrm{g}$. fibers; Reaction Temperature, $90^{\circ} \mathrm{C}$; Reaction Time, 10 min.; M:L, 1:50.

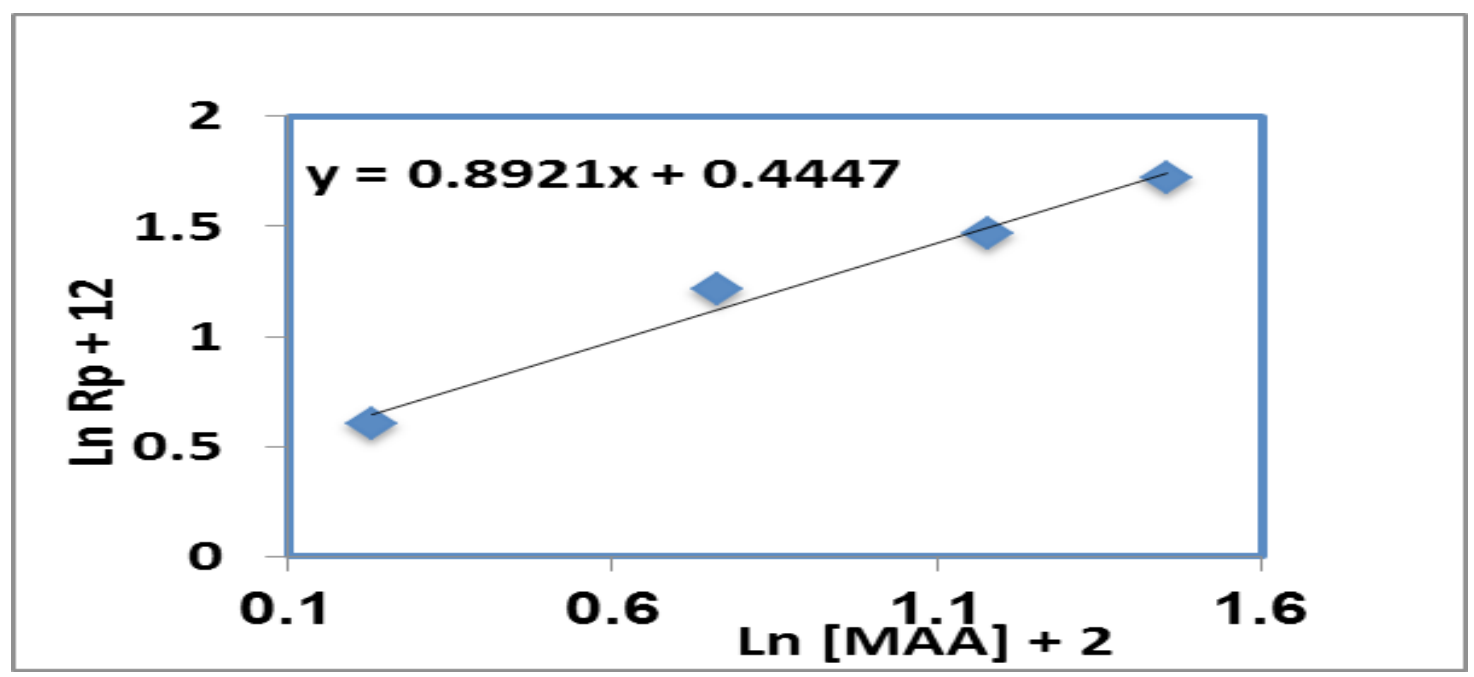

Fig. 2. Logarithmic correlation between the rate of grafting (Rp) of MAA onto PA-6 fibers on the monomer concentration

Reaction Conditions:

$\left[\mathrm{CuSO}_{4} \cdot 5 \mathrm{H}_{2} \mathrm{O}\right], 1.25 \times 10^{-3} \mathrm{~mol} / 1 ;\left[\mathrm{K}_{2} \mathrm{~S}_{2} \mathrm{O}_{8}\right], 2.20 \times 10^{-4} \mathrm{~mol} / 1 ;$; $\left.\mathrm{AS}\right], 1.63 \mathrm{mg} / \mathrm{g}$ fibers; Reaction Temperature, $90^{\circ} \mathrm{C}$; Reaction Time, 10 min.; M:L, 1:50.

TABLE 7. Effect of Reaction temperature on the rate of grafting (Rp) of MAA onto PA-6 fibers.

\begin{tabular}{|c|c|c|c|c|c|c|c|}
\hline \multirow{2}{*}{ Reaction Temperature, $\left({ }^{\circ} \mathrm{C}\right)$} & \multicolumn{5}{|c|}{ Reaction Time (min.) } & & \\
\hline & 10 & 20 & 30 & 45 & 60 & & \\
\hline 75 & 4.10 & 8.0 & 12.6 & 14.0 & 16.8 & & \\
\hline 80 & 7.30 & 10.6 & 14.8 & 17.9 & 21.2 & $0^{\circ}$ & \\
\hline 85 & 11.6 & 18.1 & 23.2 & 31.5 & 35.2 & & \\
\hline 90 & 17.8 & 28.1 & 34.9 & 44.6 & 55.0 & & \\
\hline
\end{tabular}

Reaction Conditions:

[MAA], $5.8 \times 10^{-1} \mathrm{~mol} / 1 ;\left[\mathrm{K}_{2} \mathrm{~S}_{2} \mathrm{O}_{8}\right], 2.20 \times 10^{-4} \mathrm{~mol} / 1 ;\left[\mathrm{CuSO}_{4} .5 \mathrm{H}_{2} \mathrm{O}\right], 1.25 \times 10^{-3} \mathrm{~mol} / \mathrm{l} ;[\mathrm{AS}], 1.63 \mathrm{mg} / \mathrm{g}$ fibers; M:L, 1:50.

Egypt. J. Chem. 61, No. 6 (2018) 
TABLE 8. Dependence of the rate of grafting (Rp) of MAA onto PA-6 fibers on the reaction temperature.

\begin{tabular}{|c|c|c|c|c|c|c|c|c|}
\hline \multirow{2}{*}{$\mathbf{T}$} & \multirow{2}{*}{$\mathbf{T}+273$} & \multirow{2}{*}{$1000 / T$} & \multicolumn{3}{|c|}{ Graft Yield } & \multirow{2}{*}{$\begin{array}{c}\text { Rp } \\
\text { Mol.l }^{-1} \cdot \text { sec }^{-1}\end{array}$} & \multirow{2}{*}{ Ln Rp } & \multirow{2}{*}{$\begin{array}{c}\text { Ln } \\
\text { Rp+12 }\end{array}$} \\
\hline & & & $\%$ & gr. & $\mathrm{mol} / \mathrm{l}$ & & & \\
\hline 75 & 348 & 2.87 & 4.1 & 0.020 & $4.77 \times 10^{-3}$ & $7.90 \times 10^{-6}$ & -11.74 & 0.26 \\
\hline 80 & 353 & 2.83 & 7.3 & 0.037 & $8.49 \times 10^{-3}$ & $1.41 \times 10^{-5}$ & -11.17 & 0.83 \\
\hline 85 & 358 & 2.79 & 11.6 & 0.058 & $1.35 \times 10^{-2}$ & $2.25 \times 10^{-5}$ & -10.70 & 1.30 \\
\hline 90 & 363 & 2.75 & 17.8 & 0.089 & $2.07 \times 10^{-2}$ & $3.45 \times 10^{-5}$ & -10.27 & 1.73 \\
\hline
\end{tabular}

Reaction Conditions:

[MAA], $5.8 \times 10^{-1} \mathrm{~mol} / 1 ;\left[\mathrm{K}_{2} \mathrm{~S}_{2} \mathrm{O}_{8}\right], 2.20 \times 10^{-4} \mathrm{~mol} / \mathrm{l} ;\left[\mathrm{CuSO}_{4} .5 \mathrm{H}_{2} \mathrm{O}\right], 1.25 \times 10^{-3} \mathrm{~mol} / \mathrm{l} ;[\mathrm{AS}], 1.63 \mathrm{mg} / \mathrm{g}$. fibers; Reaction Time, 10 min.; M: L, 1:50.

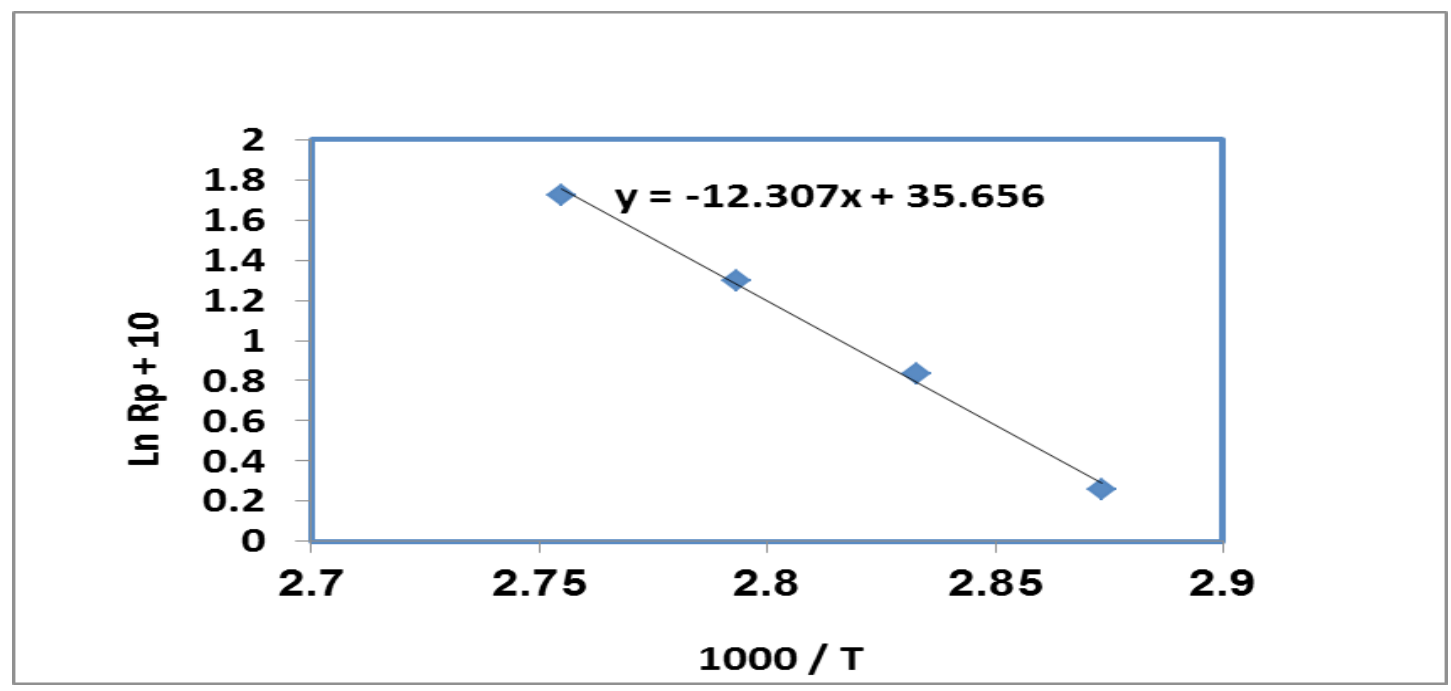

Fig.3. Arrhenius plots for the graft copolymerization of MAA onto PA-6 fibers.

$\underline{\text { Reaction Conditions: }}$

[MAA], $5.8 \times 10^{-1} \mathrm{~mol} / 1 ;\left[\mathrm{K}_{2} \mathrm{~S}_{2} \mathrm{O}_{8}\right], 2.20 \times 10^{-4} \mathrm{~mol} / 1 ;$ [CuSO$\left.{ }_{4} .5 \mathrm{H}_{2} \mathrm{O}\right], 1.25 \times 10^{-3} \mathrm{~mol} / \mathrm{l} ;[\mathrm{AS}], 1.63 \mathrm{mg} / \mathrm{g}$ fibers; Reaction Time, 10 min.; M:L, 1:50.

TABLE 9. Effect of $\mathrm{K}_{2} \mathrm{~S}_{2} \mathrm{O}_{8}$ concentration on the rate of grafting (Rp) of MAA onto PA-6 fibers.

\begin{tabular}{|c|c|c|c|c|c|c|c|}
\hline \multirow{2}{*}[\mathrm{K}_{2}\mathrm{S}_{2}\mathrm{O}_{8}]{$\times 10^{-4}, \mathrm{~mol} / \mathrm{l}$} & \multicolumn{5}{|c|}{ Reaction Time (min.) } & & \\
\hline & 10 & 20 & 30 & 45 & 60 & & \\
\hline 1.02 & 11.2 & 12.3 & 15.2 & 16.0 & 16.8 & \multirow{4}{*}{$\partial^{\circ}$} & \\
\hline 1.58 & 13.8 & 22.4 & 27.5 & 30.1 & 31.0 & & $\stackrel{3}{\stackrel{2}{2}}$ \\
\hline 1.85 & 15.5 & 25.2 & 30.1 & 35.0 & 35.5 & & 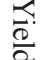 \\
\hline 2.20 & 17.8 & 28.1 & 37.2 & 48.0 & 55.0 & & \\
\hline
\end{tabular}

Reaction Conditions:

[MAA], $5.8 \times 10^{-1} \mathrm{~mol} / 1 ;\left[\mathrm{CuSO}_{4} .5 \mathrm{H}_{2} \mathrm{O}\right], 1.25 \times 10^{-3} \mathrm{~mol} / 1 ;[\mathrm{AS}], 1.63 \mathrm{mg} / \mathrm{g}$. fibers; Reaction Temperature, $90^{\circ} \mathrm{C} ; \mathrm{M}: \mathrm{L}, 1: 50$. 
TABLE 10. Dependence of the rate of grafting (Rp) of MAA onto PA-6 Fibers on the $\mathrm{K}_{2} \mathrm{~S}_{2} \mathrm{O}_{8}$ concentration.

\begin{tabular}{|c|c|c|c|c|c|c|c|c|}
\hline \multirow{2}{*}{$\begin{array}{c}{\left[\mathrm{K}_{2} \mathrm{~S}_{2} \mathbf{O}_{8}\right]} \\
\mathrm{mol} / \mathbf{l}\end{array}$} & \multirow{2}{*}{$\begin{array}{c}\mathrm{Ln} \\
{\left[\mathrm{K}_{2} \mathrm{~S}_{2} \mathrm{O}_{8}\right]}\end{array}$} & \multirow{2}{*}{$\begin{array}{c}\mathrm{Ln} \\
{\left[\mathrm{K}_{2} \mathrm{~S}_{2} \mathrm{O}_{8}\right]} \\
+\mathbf{1 0}\end{array}$} & \multicolumn{3}{|c|}{ Graft Yield } & \multirow{2}{*}{ 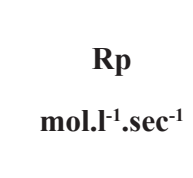 } & \multirow{2}{*}{$\begin{array}{l}\text { Ln } \\
\text { Rp }\end{array}$} & \multirow{2}{*}{$\begin{array}{c}\text { Ln } \\
\text { Rp+11 }\end{array}$} \\
\hline & & & $\%$ & gr. & $\mathrm{mol} / \mathrm{l}$ & & & \\
\hline $1.02 \times 10^{-4}$ & -9.19 & 0.81 & 11.2 & 0.056 & $1.30 \times 10^{-2}$ & $2.17 \times 10^{-5}$ & -10.74 & 0.26 \\
\hline $1.58 \times 10^{-4}$ & -8.75 & 1.25 & 13.8 & 0.069 & $1.60 \times 10^{-2}$ & $2.67 \times 10^{-5}$ & -10.53 & 0.47 \\
\hline $1.85 \times 10^{-4}$ & -8.60 & 1.40 & 15.5 & 0.078 & $1.80 \times 10^{-2}$ & $3.00 \times 10^{-5}$ & -10.41 & 0.59 \\
\hline $2.20 \times 10^{-4}$ & -8.42 & 1.58 & 17.8 & 0.089 & $2.07 \times 10^{-2}$ & $3.45 \times 10^{-5}$ & -10.27 & 0.73 \\
\hline
\end{tabular}

Reaction Conditions:

[MAA], $5.8 \times 10^{-1} \mathrm{~mol} / 1 ;\left[\mathrm{CuSO}_{4} .5 \mathrm{H}_{2} \mathrm{O}\right], 1.25 \times 10^{-3} \mathrm{~mol} / 1 ;$;AS], $1.63 \mathrm{mg} / \mathrm{g}$ fibers; Reaction Temperature, $90^{\circ} \mathrm{C}$; Reaction Time, 10 min.; M:L, 1:50.

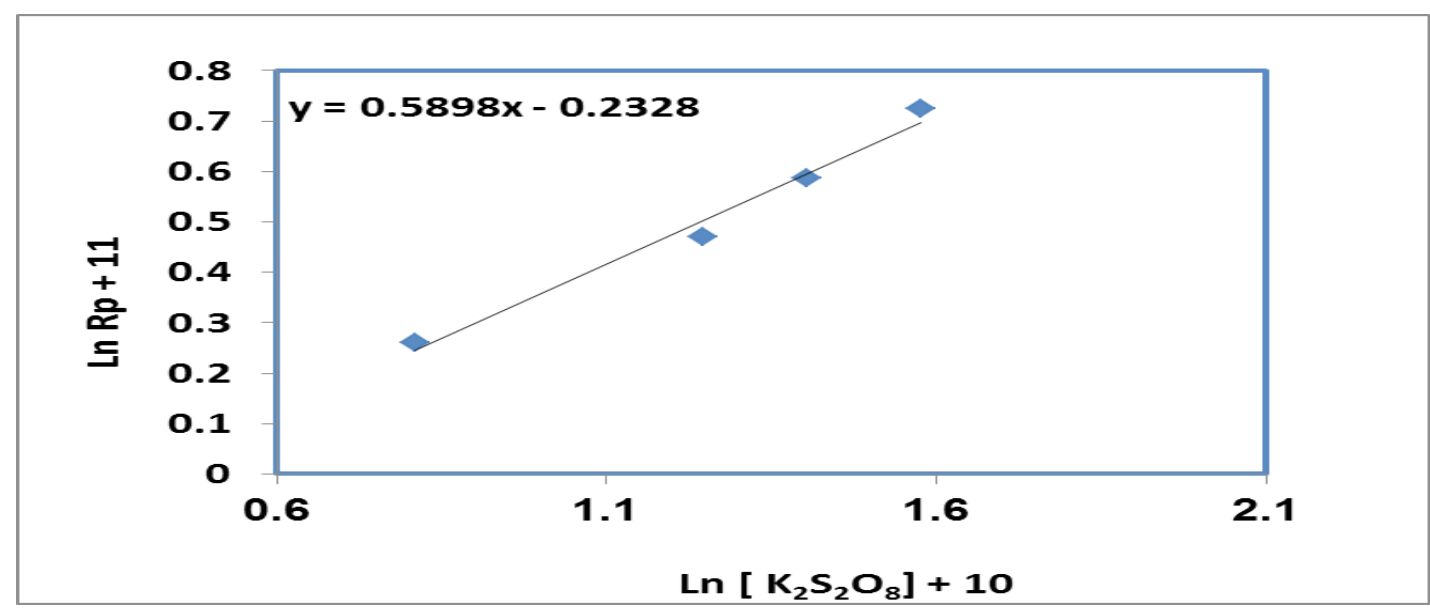

Fig. 4. Logarithmic correlation between the rate of grafting (Rp) of MAA onto PA-6 fibers on the K2S2O8 concentration.

Reaction Conditions:

[MAA], $5.8 \times 10^{-1} \mathrm{~mol} / 1 ;\left[\mathrm{CuSO}_{4} \cdot 5 \mathrm{H}_{2} \mathrm{O}\right], 1.25 \times 10^{-3} \mathrm{~mol} / 1$; $[\mathrm{AS}], 1.63 \mathrm{mg} / \mathrm{g}$ fibers; Reaction Temperature, $90^{\circ} \mathrm{C}$; Reaction Time, 10 min.; M:L, 1:50.

TABLE 11. The Effect of the Relation between the Weight of MAA and the Weight of PA-6 Fibers on the obtained Graft add-on on the Fibers.

\begin{tabular}{cc}
\hline Monomer/fiber (WT/WT) & Graft Yield \% \\
\hline $0.50 / 1$ & 28.0 \\
$0.75 / 1$ & 60.0 \\
$1: 1$ & 85.1 \\
$1.5 / 1$ & 84.3 \\
$2.0 / 1$ & 74.8 \\
$2.5 / 1$ & 69.4 \\
$3.0 / 1$ & 70.0 \\
\hline
\end{tabular}

$\underline{\text { Reaction conditions }}$

PA-6 fibers, 2g; $\left(\mathrm{K}_{2} \mathrm{~S}_{2} \mathrm{O}_{8}\right), 2.20 \times 10^{-4} \mathrm{~mol} / 1 ; \mathrm{CuSO}_{4} .5 \mathrm{H}_{2} \mathrm{O}, 1.0 \mathrm{ml}(0.05 \mathrm{M})$ Reaction Time, 60 min.; Reaction Temperature, $90{ }^{\circ} \mathrm{C}$; M: L Ratio, $1: 25$.

Egypt. J. Chem. 61, No. 6 (2018) 
The production of grafted PA-6 fibers with PMAA on pilot scale consists of scouring, treatment with AS, treatment with $\mathrm{K}_{2} \mathrm{~S}_{2} \mathrm{O}_{8}$ water solution, and finally grafting with MAA.

Scouring, treatment with AS, and treatment with persulphate solution on pilot scale were carried out in Misr El Beida company in Kafr El Dawar, Egypt. English tops machine Shell-Y42 working under pressure at maximum temperature $100^{\circ} \mathrm{C}$, volume 1000 liters and load $100 \mathrm{~kg}$ was used for carrying out the above mentioned technological processes under its optimal conditions.

Grafting the scoured, treated with AS and $\left(\mathrm{K}_{2} \mathrm{~S}_{2} \mathrm{O}_{8}\right)$ water solutions PA-6 fibers was carried out in the pilot plant of Textile Research Division in the National Research Centre in Dokki, Giza, Egypt. Pilot reactor working under pressure at maximum temperature $100^{\circ} \mathrm{C}$, volume 50 liters and load $5.0 \mathrm{~kg}$ was used for carrying out grafting reaction under its optimal conditions.

\section{Sorption Properties of PA-6 Fibers Grafted with PMAA}

The sorption properties of PA-6fibers containing AS and grafted with PMAA and the factors affecting the sorption of several metal ions $\left(\mathrm{Cu}^{2+}, \mathrm{Pb}^{2+}\right.$ and $\left.\mathrm{Cr}^{6+}\right)$ were studied. A grafted PA-6 fiber was initially prepared by polymer-analogous transformation under the action of aqueous sodium hydroxide. This treatment enhances the adsorption availability. When such sorbents are in contact with the abovementioned transition metal ions adsorption occurred with high efficiency. The adsorption mechanism of those metals ions onto the modified adsorbent could be explained by the displacement of $\mathrm{Na}^{+}$ions by $\mathrm{Cu}^{2+}, \mathrm{Pb}^{2+}$, and $\mathrm{Cr}^{6+}$ to preferentially bind with grafted fibers. Dried samples of PA-6 fibers $(0.2 \mathrm{~g})$ containing AS and grafted with PMAA and treated with sodium hydroxide solution $(0.1 \mathrm{M})$ for definite time and washed thoroughly with water thereafter were added into $100 \mathrm{~cm}^{3}$ flasks containing 60 $\mathrm{cm}^{3}$ of each metal ion solutions having constant concentration and adjusted to desired $\mathrm{pH}$. The solution was stirred under desired temperature for the desired duration. Then the solution was filtered and the ion concentration of the filtrates was analyzed. Finally, the degree of extraction $(\mathrm{R}, \%)$ and sorption capacity (SC, $\mathrm{mg} / \mathrm{g}$ ) were calculated. The effect of adsorption conditions on both (R \%) and SC (mg/g) of studied metal ions with reactive PA-6 fibers was investigated (Table 12,13).

\section{Effect of adsorption time}

Data of Table 12 represent the effect of adsorption time on the degree of extraction $\mathrm{R} \%$ and sorption capacity of PMAA grafted nylon fiber in salt form. It was found that at short time (10 minutes) there was a significant increase in $\mathrm{R}$ $\%$ and $\mathrm{SC}, \mathrm{mg} / \mathrm{g}(78.3,47.8,15.5)$ and $\mathrm{SC}(18.8$, $11.5,0.40)$ in case of sorption of $\mathrm{Cu}^{2+}, \mathrm{Pb}^{2+}$ and $\mathrm{Cr}^{6+}$ respectively. Increasing the adsorption time up to 20 minutes, led to increase in the degree of extraction and sorption capacity. Further increase in the adsorption time has no effect on the aforementioned parameters i.e. the adsorption equilibrium reached within 20-30 minutes of the adsorption process. It was noticed that the order of sorption capacity of the metal ion used is in the following order: $\mathrm{Cu}^{2+}>\mathrm{Pb}^{2+}>\mathrm{Cr}^{6+}$.

\section{Effect of initial ion concentration}

Table 12 showed the effect of initial concentrations of the metal ion on $\mathrm{R} \%$ and $\mathrm{SC}$ respectively. It was found that the adsorption of metal ions increased with increasing initial ion concentration, then reached a plateau value at higher concentration. This may be attributed to the saturation of the reactive sites available for the adsorption of the metal ions.

\section{Effect of Temperature}

The effect of temperature on the adsorption of metal ions onto salt form of grafted PA-6 fibers was studied within the range $30-80^{\circ} \mathrm{C}$. The results are shown in Table 12. It was noticed that increasing the temperature from $30-80^{\circ} \mathrm{C}$ increased the adsorption amount of metals ions which could be attributed to increasing of the swelling degree of reactive fibers. The percentage of swelling increases as the temperature increase due to increasing the flexibility of the grafted fibers chains. The degree of extraction and the sorption capacity of both $\mathrm{Cu}^{+2}$ and $\mathrm{pb}^{+2}$ ions increases, whereas a little effect on the adsorption of $\mathrm{Cr}^{+6}$ ions was noticed.

\section{Effect of the $p H$}

Data present in Table 13 clarify the effect of $\mathrm{pH}$ on the adsorption of metal ions $\mathrm{R} \%$ and $\mathrm{SC}$, $\mathrm{mg} / \mathrm{g}$ values. It was noticed that, both $\mathrm{R} \%$ and $\mathrm{SC}$ values decrease with decreasing $\mathrm{pH}$ values. This can be attributed to the increasing of $\mathrm{H}^{+}$ions at the surface of fibers which repel the metal ions [32]. The net result is directed to decrease $\mathrm{R} \%$ and SC values. The data listed in Table 13 also pointed to the increase of $\mathrm{pH}$ values lead to increase $\mathrm{R} \%$ and $\mathrm{SC}$ values. 
TABLE 12. Effect of Adsorption time (min.), Initial Concentration of Metal Ions and Adsorption Temperature $\left({ }^{\circ} \mathrm{C}\right)$ on the Degree of Extraction (R\%) and the Adsorption Capacity (SC, mg/g) of Metal Ions with PA-6 Fibers Treated with AS and Grafted with PMAA.

\begin{tabular}{|c|c|c|c|c|c|c|c|c|c|c|}
\hline \multirow{3}{*}{$\begin{array}{l}\text { Metal } \\
\text { Ions }\end{array}$} & \multicolumn{5}{|c|}{ Degree of Extraction (R \%) } & \multicolumn{5}{|c|}{ Adsorption Capacity (SC, mg/g) } \\
\hline & \multicolumn{10}{|c|}{ Adsorption Time, ( minutes) ${ }^{a}$} \\
\hline & 10 & 20 & 30 & 60 & 120 & 10 & 20 & 30 & 60 & 120 \\
\hline $\mathrm{Cu}^{2+}$ & 78.3 & 86.0 & 86.0 & 86.0 & 86.0 & 18.8 & 20.6 & 20.6 & 20.6 & 20.6 \\
\hline $\mathrm{Pb}^{2+}$ & 47.8 & 85.4 & 85.4 & 85.4 & 85.4 & 11.5 & 20.5 & 20.5 & 20.5 & 20.5 \\
\hline $\mathrm{Cr}^{6+}$ & 15.5 & 32.0 & 32.0 & 32.0 & 32.0 & 0.40 & 9.56 & 9.56 & 9.56 & 9.56 \\
\hline Metal & \multicolumn{10}{|c|}{ Initial concentration, $(\mathrm{ppm})^{\mathrm{b}}$} \\
\hline Ions & 60 & 80 & 100 & & 200 & 60 & 80 & 100 & & 200 \\
\hline $\mathrm{Cu}^{2+}$ & 49.5 & 86.0 & 86.0 & & 86.0 & 19.3 & 20.6 & 20.6 & & 20.6 \\
\hline $\mathrm{Pb}^{2+}$ & 79.3 & 85.4 & 85.4 & & 85.4 & 24.2 & 20.5 & 20.5 & & 23.8 \\
\hline $\mathrm{Cr}^{6+}$ & 26.6 & 32.0 & 32.0 & & 32.0 & 8.0 & 9.60 & 9.60 & & 9.40 \\
\hline Metal & \multicolumn{10}{|c|}{ Adsorption Temperature, $\left({ }^{\circ} \mathrm{C}\right)^{\mathrm{c}}$} \\
\hline Ions & 30 & 40 & 60 & & 80 & 30 & 40 & 60 & & 80 \\
\hline $\mathrm{Cu}^{2+}$ & 86.0 & 95.4 & 99.2 & & 99.7 & 20.6 & 22.9 & 23.8 & & 24.0 \\
\hline $\mathrm{Pb}^{2+}$ & 85.4 & 87.2 & 88.4 & & 91.2 & 20.5 & 21.0 & 21.2 & & 21.9 \\
\hline $\mathrm{Cr}^{6+}$ & 32.0 & 32.5 & 32.8 & & 34.4 & 9.6 & 9.2 & 9.6 & & 9.60 \\
\hline
\end{tabular}

Adsorption Conditions:

${ }^{a}$ Initial concentration of Metal Ions, $80 \mathrm{ppm}$; Adsorption Temperature, $30^{\circ} \mathrm{C} .{ }^{\mathrm{b}}$ Adsorption Time, $1 \mathrm{hr}$; Adsorption Temperature, $30^{\circ} \mathrm{C}$.

' Initial concentration of Metal Ions, $80 \mathrm{ppm}$; Adsorption Time, $1 \mathrm{hr}$;

abc Adsorption pH, 6.0; [AS], 1.63 mg/gm; Graft Yield, $30 \%$, (Salt Form).

TABLE 13. Effect of pH on the Degree of Extraction (R \%) and the Adsorption Capacity (SC, mg/g) of Metal Ions with PA-6 Fibers Treated with AS and Grafted with PMAA.

\begin{tabular}{|c|c|c|c|c|c|c|c|}
\hline \multirow{2}{*}{$\begin{array}{l}\text { Metal } \\
\text { Ions }\end{array}$} & \multirow{2}{*}{ pH } & \multicolumn{3}{|c|}{ Degree of Extraction (R \%) } & \multicolumn{3}{|c|}{ Adsorption Capacity (SC, mg/g) } \\
\hline & & Nylon-6 & $\begin{array}{c}\text { Nylon-6 } \\
\rightarrow \text { AS }\end{array}$ & $\begin{aligned} & \text { Nylon-6 } \rightarrow \text { AS } \\
& \rightarrow \text { PMAA }(30 \%)\end{aligned}$ & Nylon-6 & $\begin{array}{l}\text { Nylon-6 } \\
\rightarrow \text { AS }\end{array}$ & $\begin{array}{c}\text { Nylon-6 } \rightarrow \text { AS } \rightarrow \\
\text { PMAA }(30 \%)\end{array}$ \\
\hline \multirow{3}{*}{$\mathrm{Cu}^{2+}$} & 4 & 39.8 & 47.8 & 71.1 & 9.5 & 11.5 & 16.2 \\
\hline & 6 & 46.3 & 53.6 & 86.0 & 11.1 & 16.6 & 20.6 \\
\hline & 6.5 & 48.3 & 69.4 & 86.0 & 11.6 & 17.9 & 17.1 \\
\hline \multirow{3}{*}{$\mathrm{Pb}^{2+}$} & 4 & 18.6 & 24.9 & 34.2 & 4.50 & 6.2 & 16.5 \\
\hline & 6 & 44.6 & 56.4 & 85.4 & 10.7 & 7.1 & 20.5 \\
\hline & 6.5 & 58.4 & 61.8 & 86.0 & 14.0 & 14.8 & 22.2 \\
\hline \multirow{3}{*}{$\mathrm{Cr}^{6+}$} & 4 & 26.3 & 27.5 & 32.0 & 6.3 & 7.6 & 9.6 \\
\hline & 6 & 26.9 & 29.5 & 32.0 & 7.5 & 8.6 & 9.6 \\
\hline & 6.5 & 30.6 & 32.3 & 33.4 & 8.5 & 9.0 & 15.8 \\
\hline
\end{tabular}

Adsorption Conditions:

Time of Adsorption, $1 \mathrm{hr}$; Adsorption Temperature, $30^{\circ} \mathrm{C}$; [AS],

$1.63 \mathrm{mg} / \mathrm{gm}$; (Salt Form). 


\section{Conclusion}

Grafting of MAA, with high rate, and without homopolymer formation, on PA-6 fibers for ion exchange application was carried. Factors affecting the amount of PMAA has been investigated. The optimum conditions for grafting reaction on laboratory scale were adjusted and adapted to suit the production of grafted PA-6 fibers on pilot scale. Assessment the application of the produced fibers as ion-exchanger was evaluated for adsorption of heavy metals. The grafted fibers showed high efficiency for adsorption of $\mathrm{Cu}^{2+}$, $\mathrm{Pb}^{2+}$ and $\mathrm{Cr}^{6+}$. The effect of adsorption parameters such as $\mathrm{pH}$, duration, initial ion concentration and the adsorption temperature on the degree of extraction (R \%) and adsorption capacity (SC, $\mathrm{mg} / \mathrm{g}$ ) was calculated. The results revealed that, the adsorption of these selected metals in the following order:

$$
\mathrm{Cu}^{2+}>\mathrm{Pb}^{2+}>\mathrm{Cr}^{6+} \text {. }
$$

\section{Acknowledgment}

The authors of this article would like to thank the Science and Technology Development Foundation (STDF) - Egypt, for funding the project ID 2723.

\section{References}

1. Naumenko, E.A.; Danilova, E. Ya.; Emets, L.V.; V01'f, L. A.; Antokol'skaya, I. I.; Myasoedova, G. V., Sorbent fibers which are selective to platinum group metals and gold, Khim Volokna, 17 (1), 6667 (1985).

2. Hayakawa, K.; Yamakita, H., Preparation of mercaptoethylated graft fibers for adsorption of heavy metal ions, J Appl Polym Sci 21 (3), 665675 (1977).

3. Timus, D. M.; Popov, A. M. X., Some preliminary studies of the thermal behaviour of electron beam grafted polyamides. Appl Radiat Isot 48, 16571662 (1977).

4. Kato, K.; Uchida, E. T.; Kang, Y.; Ikada, Y., Polymer surface with graft chains, Progress in Polymer Science, 28, 209-259 (2003).

5. Lysenko, A. A.; Prisekina, O.V.; Nemilov V. E; Kazakevich Yu. E, Bytsan N. V.; and Danilova, E. Ya., Carbochain ion-exchange fibers prepared by the graft polymerization method, Khim Volokna, 19 (1), 20-24 (1987).

6. Lavnikova, I. V.; Zheltobryukhov, V. F., Radical graft polymerization of glycidyl methacrylate and polycaproamide fiber, Russian Journal of Applied Chemistry, 74 (4), 678-3680 (2001).

7. Soldatov, V. S.; Shunkevich, A. A.; Sergeev, G. I., Synthesis, structure and properties of new fibrous ion exchangers, Reactive Polymers, 7, 159 (1988).

8. Solovey, O. M.; Shunkevich, A. A.; Pokrovskaya, A.I.; Elinson, I. S.; Johann; J.; Iraushek, H., Chemically active textile materials as efficient means for water purification. Desalination 124, 182-192 (1999).

9. Lin, W.P.; Lu, Y.; Zeng, H.M., Preparation of the amidoxime group containing chelating fiber and adsorption property of gold. I. Preparation of chelating fiber by amidoximation of polyacrylonitrile fiber Reactive. Polymers, 17, 155-161 (1992).

10. Lu, Y.; Zhang, Z.; Zeng, H., Studies on preparation and ion-exchange properties of weakly acidic cation exchange fiber by preirradiation-induced graft copolymerization with electron beam. $J$. Appl Polym Sci, 53, 405 (1994).

11. Druzhinina, T.V.; Nazarina, L. A., Chemisorptions fibers based on graft copolymers: fabrication and properties. A review, Khim Volokna 31 (4), 252262 (1999).

12. Druzhinina, T.V.; Struganova, M. A., Fabrication of polycaproamide sorption fibers with hydrazine group, Fiber Chemistry, 33(1), 5 (2001).

13. Abaldueva, E.V.; Druzhinina, T.V., Sorption of heavy metals by polyamide chemisorption fiber, Fiber Chemistry, 36 (1), 33 (2004).

14. Shalaby, S.E; Al- Balakocy, N.G. Egypt Pat 941 (2006). A simple efficient and generally applicable method For grafting of vinyl monomers onto nylon-6 fabrics, Egypt. Pat. No. 23507 (2006).

15. Shalaby, S. E; Abdalla, N. H.; Rabie, A.M. 2- 4 Mar. Chemical modification of polyamide- 6 fibers containing chemically bonded quaternary ammonium groups with 2-dimethylaminoethlmethacrylate. $1^{\text {st }}$ International Conference, Textile Research Division NRC, Cairo, Egypt (2004).

16. Gabrielyan G. A; Shalaby S. E; Druzhinina T.V.; Rogovin Z. A.; Afanaceva I. S., Modification of polycaproamide fibers. U.S.S.R. SU 907, 111(Cl. D06 M13/46), 23 Feb. (1982).

17. Gabrielyan G. A; Shalaby S. E.; Druzhinina T.V.; Rogovin Z. A., Modification of polycaproamide fibers. U.S.S.R. SU 891, 821(CI. D06M13/46), 23

Egypt. J. Chem. 61, No. 6 (2018) 
Dec. (1981).

18. Gabrielyan G. A; Shalaby S. E.; Druzhinina T.V.; Rogovin Z. A., Graft copolymers U.S.S.R. SU 914, 677 (Cl. D01F11/04), 23 March (1982).

19. Shalaby S. E.; Gabrielyan G. A; Rogovin Z. A. Synthesis of graft copolymers of polycaprolactam with poly Dimethylamino ethyl methacrylate using the potassium persulphate $-\mathrm{Cu} 2+$ ion initiating system. Vysokomol. Soedin., Ser. B, 24(4), 288 (1982); Chem. Abstr. 97 (6), 39490n (1982).

20. Shalaby, S. E.; Afamaceva, I. S.; Gabrielyan, G. A.; Druzhinina, T.V., Sorption and electrostatic properties of polycaprolactam fibers modified by polyacrylonitrile grafting. Khim. Volokna, (2), 30 (1982).

21. Shalaby, S.E.; Piankova, A. B.; Gabrielyan, G. A.; Druzhinina, T.V.; Rogovin, Z. A. (1982). Synthesis of graft polycaproamide copolymers using binary mixtures of monomers, Khim. Volokna, (3), 10; Chem. Abstr. 97, (10), 73780s (1982).

22. Shalaby, S. E.; Afanaceva, I. S.; Gabrielyan, G. A.; Druzhinina, T. V.; Rogovin, Z. A., Synthesis of graft copolymers of polycaprolactam with poly(Dimethylamino ethyl methacrylate) using the potassium persulphate-Cu2+ ion initiating system. Vysokomol. Soedin., Ser. B, 24(4), 288 (1982).; Chem. Abstr. 97 (6), 39490n (1982).

23. Shalaby, S.E.; Gabrielyan, G.A.; Druzhinina, T.V.; Rogovin, Z. A. Chernukhina, A. I., Method for the preparation of multi-graft copolymers of polyamide-6 fibers. U.S.S.R. SU 103, 1971(cl. D01F11/04), 1 April. (1983).

24. Shalaby, S. E.; Afanaceva, I. S; Gabrielyan, G. A; Druzhinina, T. V., Studies of properties of chemically modified polycaproamide fibers via grafting with polyacrylonitrile. Khim. Volokna, (3), 32 (1984).

25. Metwally, R. N.; El- Shahid M. F.; Shalaby, S. E.; Abdelhay, A. Amination of Polyamide fibers grafted with polyglycidylmethacrylate. Part 1:
Factors affecting the amination reaction using different amines. Procedure of the First Egyptian Syrian Conference in Chemical Engineering, Faculty Of Petroleum and Mining Engineering, Suez, P138 (1995).

26. Metwally, R. N.; El- Shahid M. F.; Shalaby, S. E.; Abdelhay. A. Amination of polyamide fibers grafted with polyglycidylmethacrylate. part: Properties of aminated of polyamide fibers grafted with polyglycidylmethacrylate. Procedure of the First Egyptian Syrian Conference in Chemical Engineering, Faculty of Petroleum and Mining Engineering, Suez, P.162 (1995).

27. Shalaby, S. E.; Al- Balakocy, N. G; Abo El-Ola, S. M. Graftcopolymerization of glycidylmethacrylate onto modified nylon-6 fibers, J Appl Polym Sci., 99, 613- 618 (2006).

28. Shalaby, S. E.; Al-Balakocy, N. G.; Abo El-Ola, S. M., Surface modification of nylon-6 fibers for medical application, J. Appl Polym Sci, 104, 37883796 (2007).

29. Shalaby, S. E.; Al-Balakocy, N. G; Abdel-Fatah, O.M.; Elshafei, A. M., Antimicrobial finishing of regular and modified nylon-6 fabrics. J. Appl Polym Sci, 110 (2), 738 (2008).

30. Al-Balakocy, N. G., Studies on some chemical modifications of polycaprolactam fibers Ph.D. Dissertation, Ain-Shams University, Cairo, Egypt, (2002).

31. Shalaby, S. E.; Al-Balakocy; Beliakova, M. K., A simple efficient and generally applicable method for imparting polyester and polyester/cotton fabrics antimicrobial activity, Egypt. Pat. No. 24672 (2010).

32. Shalaby, S. E; Al-Balakocy N. G; Beliakova, M. K.; Simple method for grafting of glycidyl methacrylate onto nylon-6 nonwoven fabrics for ion exchange applications, International Journal of Science and Research, 4, 689-696 (2015).

(Received 23/4/2018; accepted 2/8/2018) 
طريقة مطورة لإنتاج ألياف البولي اميد ــ المطعة بحامض البولي ميثاكريليك على مستوى نصف صناعى وتطبيقها كمبادل أيونى عامى السباعى شلبى، سميحة محمد ابو العلا، ناصر جاد احمد البلاكوسى، مارجاريتا بيلاكوفا، رافت عبده شعبة بحوث الصناعات النسجية ـ المركز القومى للبحوث ـ سب شارع البحوث (التحرير سابقا)، الدقى، الجيزة-

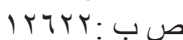

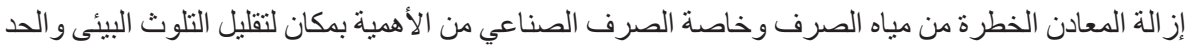

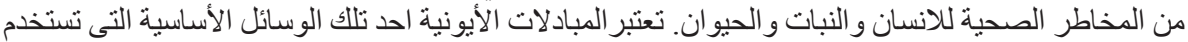

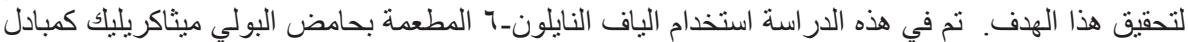

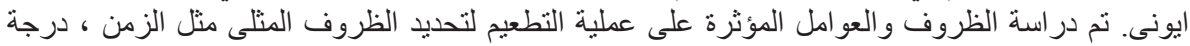

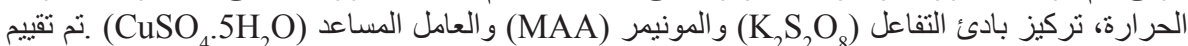

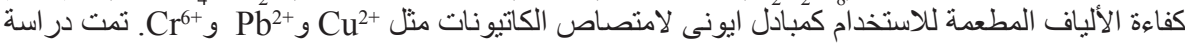

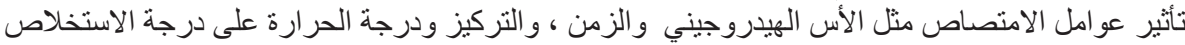

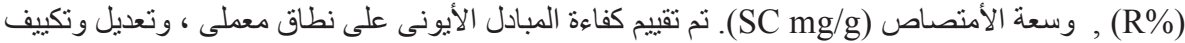
هذه الثروط لتتناسب مع إنتاج هذه الألياف المطعمة على نطاق تجريبي. 